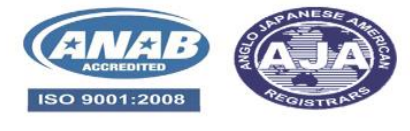

\title{
An update in the etiopathogenesis of striae distensae: A review article.
}

\author{
Aya Ahmed Al-shandawely ${ }^{1}$, Reham Ezz Eldawla ${ }^{1}$, Fatma El_Zahraa Salah \\ El_Deen Yassin ${ }^{2}$, Soha Aboeldahab ${ }^{1}$
}

1- Dermatology, Venereology \& Andrology Department, Faculty of Medicine, Sohag University.

2- Pathology Department, Faculty of Medicine, Sohag University

\begin{abstract}
:
Striae distensae (SD), also known as stretch marks, are common skin lesions causing significant psychological stress and cosmetic disfigurement. They are common to be seen during pregnancy, rapid weight change, puberty, many other pathological conditions including Cushing disease, anorexia nervosa, even excessive use of certain drugs. Clinically, they appear as either red raised linear bands known as striae rubrae (SR) or white depressed atrophic bands known as striae alba (SA). They were first described histopathologically in 1889. Many theories have been established to explain the etiopathogenesis of SD, yet the exact mechanism is still unclear. Also, many treatment modalities have been applied to reach maximum efficacy with the least side effects. This review article aims to optimize the most recent and accepted concepts on etiopathogenesis and pathophysiology of SD which may help in finding the best lines of management with the most satisfactory outcomes.
\end{abstract}

Keywords: striae distensae, stretch marks, etiopathogenesis.

\section{Introduction:}

Striae distensae (SD) represent a major dermatological complaint, although they aren't considered as a medical emergenc$y$, they are often associated with psychological morbidity with a negative impact on quality of life in affected patients ${ }^{(1)}$.

Striae distensae are mainly caused by marked stretching in the skin leading to dermal damage and epidermal thinning, so presented clinically as linear atrophic scars. Nardelli called those lesions striae atrophic in $1936^{(2)}$.

Prevalence of SD is up to $90 \%$ of the general population, with being more common among female adolescents than males. A higher incidence of up to $90 \%$ was found in pregnant females, which are called striae gravidarum $(\mathrm{SG})^{(3)}$.

Clinical presentation of SD varies according to the stage of maturation. They appear early as erythematous raised linear lesions that may be associated with mild itching, called striae rubrae (SR). With further maturation, atrophy is established and lesions appear as white depressed scars which are called striae alba (SA). Less common types of SD include striae cerulean appearing as blue lines and striae nigral appearing as brown-black lines ${ }^{(4)}$.

The anatomical sites of SD vary and may give an idea about the underlying etiology of SD. In cases of pubertal growth, thighs, buttocks, and lumbosacral 
areas are more commonly affected. In $\mathrm{SG}$, the abdomen, breasts, and thighs are the main sites to be affected. In steroidinduced SD, large and long SR is usually found in the extremities and abdomen ${ }^{(5)}$.

\section{Etiology of SD:}

Many different risk factors were claimed to be the cause of SD development. The most common causes include a growth spurt during puberty; this explains the high prevalence of SD among adolescents. Also, pregnancy, positive family history, obesity, and rapid weight gain or loss are considered as leading causes for SD ${ }^{(6)}$. Genetic causes including Marfan syndrome, Ehler-Danlos syndrome, and ectodermal dysplasia ${ }^{(7)}$.

Endocrinal abnormalities as in cases of Cushing disease, variations in the elastic fiber component of the skin extracellular matrix contribute to the development of stretch marks. Anorexia nervosa, infections including TB and typhoid may be due to release of what is called striae toxin, chronic liver illness, and Iatrogenic causes including long-term use of certain drugs such as corticosteroids, anti-retroviral drugs used in the treatment of AIDS. All of those causes are associated with stretching of the skin leading to pathological changes in $\mathrm{SD}^{(8)}$.

\section{Pathogenesis of SD:}

There is a true debate about the definite pathogenesis of SD and this controversy led researchers to consider SD as a multifactorial condition. Defective developent of the skin with improper elastic tissue growth, abnormal stretching of the skin, and endocrinal imbalance is believed to be the predominant mediators for SD development ${ }^{(9)}$.

1) Dermal and connective tissue changes On describing the pathogenesis of SD in detail, it would be clear that stages of SD are nearly the same as stages of scar for- mation. It all begins with over-stretching of the skin leading to tears and damage in the dermis with inflammatory edema causing changes in extracellular matrix (ECM) components including rupture in collagen, elastin, and fibronectin fibers (10). Recently, it was found that dermal changes are associated with a defect in fibroblasts' function, a decrease in fibrillin and elastin, and lack of organization of collagen fibrils into bundles causing atrophic changes in $\mathrm{SD}^{(11)}$.

It was revealed that weakness in the connective tissue and loss of its elasticity is the main leading causes in the pathogennesis of SD. This was explained in the $r-$ ecent studies that found a strong association between the development of SD and pelvic organ prolapse ${ }^{(12)}$ and the association between severity of SG and development of perineal tears during labor ${ }^{(13)}$.

2) Genetic factors:

As regards the SD gene expression results; the most evident expressed genes were the secretoglobulins (SCGBs) which mainly interact with some steroid hormones. The anti-inflammatory effect of steroids is mediated mainly by the SCGBs. This may explain the strong association between the appearance of SD and the prolonged use of steroids or diseases such as Cushing syndrome in which the steroid's metabolism is affected ${ }^{(14)}$.

Keratin genes are negatively regulated in SD. Keratins are part of the cytoskeleton of epithelial cells that anchor epithelial cells and adjacent tissues providing suport during mechanical stress, this indicates that there is a loss of the mechanobiological power in the skin affected with $\mathrm{SD}^{(15)}$.

\section{3) Hormonal factors:}

Striae distensae have been directly related to hormonal changes. It was found that the expression of estrogen, androgen, and glucocorticoids receptors within SD 
is significantly higher than that of normal skin. This leads to an increase in glucocorticoids activity which suppresses the activity and proliferation of fibroblasts, $t$ hus there is a marked reduction in the formation of collagen and elastic fibers withdefects in connective tissue repair ${ }^{(16)}$. Relaxin hormone shows about a ten-fold increase during pregnancy to prevent uterine contractions till late pregnancy. It also decreases the level of collagen through inhibition of collagen density and organization. This is correlated to the increased incidence of SG due to decrease$\mathrm{d}$ ability of the skin to withstand mechanical stress ${ }^{(17)}$. Recent studies consider SD as scars. Repair of the inflammatory changes occurs under tensile forces leading to the formation of linear stretch marks perpendicular to the direction of the skin tension ${ }^{(18)}$.

4) Vitamin deficiency:

The role of vitamin D deficiency in the development of SD has been discussed in recent researches which revealed that normal values of serum 25-hydroxyvitamin D are linked to a lower risk of SD development when compared to low serum levels ${ }^{(19)}$.

This can be explained by the role of the active form of vitamin $\mathrm{D}$ in maintaining the epidermal permeability barrier and induction of fibroblasts and keratinocytes proliferation and activity ${ }^{(20)}$.

\section{Histopathology of SD:}

The first histopathological description of $\mathrm{SD}$ was in 1889. The main changes include degranulation of the mast cells in association with mid-dermal elastolysis ${ }^{(21)}$. There is also evident atrophy of the epidermis in association with flattening of the rete ridges. Dermis show thinning with a densely packed region of collagen and elastic fibers and reorganization of dermal ground substance ${ }^{(22)}$.
Using reflectance confocal microscopy in SD examination, researchers observed distortion in the papillary dermis with less coarse collagen than that found in the reticular dermis which revealed the fact that collagen in striae rubrae is finer than that in striae alba ${ }^{(23)}$.

\section{Dermoscopic features of SD:}

Several dermoscopic changes are observed in SD according to their type. The honeycomb melanotic network of the normal skin is always altered in SD. Dermoscopy shows a whitish foggy aspect with only discrete structures in striae alba while in striae rubrae, it shows a faint streaky pattern of dilated vessels orientated at a right angle to the axis of the striae, striae cerulean shows transversal bluish striations, and striae nigral are characterized by a prominent streaky melanotic pattern in continuity with the honeycomb melanotic network of the adjacent normal skin ${ }^{(24)}$.

\section{Anisotropic properties of SD:}

A study was done by Kim, Jung and Kim (2020), revealed that SD is significantly more anisotropic than the adjacent normal skin as regard both texture (as the skin surface is elongated directionally along with the physical stretching of the inner structure of SD) and viscoelasticity (as SD are less elongated by negative pressure and less retracted when relaxed meaning that SD is less viscoelastic than the normal skin) ${ }^{(25)}$.

\section{Differential diagnosis of SD:}

Striae rubrae may be mistakenly reported as child abuse especially when presented in suspected areas such as the back, abdomen, and limbs. Linear focal elastosis is another important differential diagnosis that is characterized by several asymptomatic, yellow, palpable, irregularly 
indurated, striae-like lines extending horizontally across the middle and lower ${ }^{(26)}$.

\section{Treatment of SD:}

Although many modalities have been applied for the treatment of SD, none showed a consistent efficacy or was considered as a gold standard line for management. This makes SD a highly challenging problem and a target for further studies and researches ${ }^{(27)}$.

Treatment lines include topical therapy such as topical retinoids, emollients, salicylic acid, chemical peels including trichloroacetic acid (TCA), microdermabrasion, intradermal injection of plateletrich plasma (PRP) or carboxytherapy, light therapy including ablative, non-ablative laser therapy, and intense pulsed light therapy (IPL), radiofrequency, and filler injection ${ }^{(28)}$.

\section{Conclusion:}

Striae distensae are very common skin lesions, considered as rich media for further researches and clinical trials to understand the exact etiopathogenesis and pathophysiology which would help in reaching the optimum lines of treatment with the maximum efficacy and the least side effects. Skin overstretching with decrease elasticity and endocrinal abnormalities is considered as the leading mechanisms for the development of SD.

\footnotetext{
Abbreviations:

ECM: Extracellular matrix.

SA: Striae albae.

SCGBs: Secretoglobulins.

SD: Striae distensae.

SG: Striae gravidarum.

SR: Striae rubrae.
}

\section{References:}

1- Bogdan C, Iberian S, Tomita I and Moldovan L: Improvement of skin condition in striae distensae: development, characterization and clinical efficacy of a cosmetic product containing Punica granatum seed oil and Croton lechleri resin extract. Drug Design, Development and Therapy. 2017; 11(1): 521-531.

2- Heba Elsedfy: Striae distensae in adolescents: a mini-review. Acta Biomed. 2020; 91(1): 176-181.

3- El-Domyati M, Hosam El-Din W, Medhat W, Ibrahim M, and Khaled Y: Carboxytherapy for striae distensae: a promising modality. Journal of Cosmetic Dermatology. 2021; 20(2):546-553

4- Ud-Din S, McGeorge D, and Bayat A: Topical management of striae distensae (stretch marks): prevention and therapy of striae rubrae and albae. Journal of the European academy of dermatology and venereology. 2016; 30(2):211-222.

5- Keen MA: Striae distensae: what's new at the horizon?. British Journal of Medical Practitioners. 2016; 9(3):a919.

6- Sawetz I, Lebo B, Nischwitz P, Winter R, Schaunig C, Brinskelle P, and Lumenta B: Platelet-rich plasma for striae distensae: What do we know about processed autologous blood contents for treating skin stretchmarks?-A systematic review. International Wound Journal. 2021; 18(3): 387-395.

7- Guertler A, Reinholz M, Steckmeier S and Gauglitz GG: Evaluation of a non-ablative, fractional $1565 \mathrm{~nm}$ laser for the improvement of striae distensae albae. Journal of the European Academy of Dermatology and Venereology. 2019;33(1):220-226.

8- Strumia R: Skin signs in anorexia nervosa. Dermato-endocrinology. 2009; 1(5):268-270.

9- El-Domyati M, Hosam El-Din W, Medhat W, Ibrahim MR, and Khaled Y: Carboxytherapy for striae distensae: A promising modality. Journal of 
Cosmetic Dermatology. 2021;20(2):546553.

10- Youssef S, El-Khateeb E, Aly D, and Moussa M: Striae distensae: Immunohistochemical assessment of hormone receptors in multigravida and nulligravida. Journal of Cosmetic Dermatology. 2017; 16(2):279-286.

11- Yu Y, Wu H, Yin H, and Lu Q: Striae gravidarum and different modalities of therapy: a review and update. Journal of Dermatological Treatment. 2020; 30(2):1-9.

12- Miranne J, Kramer M, Mete $M$ and Iglesia C: The Association of Abdominal Striae with Pelvic Organ Prolapse. Female Pelvic Medicine and Reconstructive Surgery. 2019; 25(4): 305-308.

13- Banu M, Nargis S, Rahman M, Sina M, Pervin M, and Manjari M: Prediction of Perineal Tear During Childbirth by the Assessment of Striae Gravidarum Score. Medicine Today. 2019; 31(2):64-67.

14-Schuck D, de Carvalho C, Sousa M, Fávero P, Martin A, Lorencini M, and Brohem C: Unraveling the molecular and cellular mechanisms of stretch marks. Journal of Cosmetic Dermatology. 2020; 19(1):190-198.

15-McLellan J, Thornhill SG, Shelton S and Kumar M: Keratin-based biofilms, hydrogels, and biofibers. InKeratin as a Protein Biopolymer. Springer, Cham. 2019; chapter 7: 187200.

16-Farahnik B, Park K, Kroumpouzos G, and Murase J: Striae gravidarum: Risk factors, prevention, and management. International Journal of Women's Dermatology. 2016; 3(2):77-85.

17-Kokanalı D and Çağlar A: Hidden association between the presence and severity of striae gravidarum and low back pain in pregnancy. European
Journal of Obstetrics and Gynecology Reproductive Biology. 2019; 233: 4952.

18-Cordeiro R, Zecchin K, and de Moraes A: Expression of estrogen, androgen, and glucocorticoid receptors in recent striae distensae. International Journal of Dermatology. 2010; 49(1):30-32.

19- Hocaoglu E, Hocaoglu M, and Akdeniz E: Association between serum 25-hydroxyvitamin D levels and the presence and severity of striae gravidarum in primigravid women. Journal of Cosmetic Dermatology. 2020; 19(11):3107-3114.

20- Cho Y, Seo C, Joo S, Song J, Cha E, and Ohn S: The association between postburn vitamin $\mathrm{D}$ deficiency and the biomechanical properties of hypertrophic scars. Journal of Burn Care Research. 2019; 40(3): 274-280.

21- Al-Himdani S, Ud-Din S, Gilmore S and Bayat A: Striae distensae: a comprehensive review and evidencebased evaluation of prophylaxis and treatment. British Journal of Dermatology. 2014; 170(3): 527-547.

22- Hengge U, Ruzicka T, Schwartz R, Cork M: Adverse effects of topical glucocorticosteroids. Journal of the American Academy of Dermatology. 2006; 54(1):1-5.

23- Rolfe H, Wurm E, and Gilmore S: An investigation of striae distensae using reflectance confocal microscopy. Australasian Journal of Dermatology. 2012; 53(3):181-185.

24- Hermanns $\mathbf{J}$ and Pierard G: Highresolution epiluminescence colorimetry of striae distensae. Journal of the European Academy of Dermatology and Venereology. 2006; 20(3): 282-287.

25- Kim M, Jung Y, and Kim E: Evaluation of anisotropic properties of striae distensae with regard to skin surface texture and viscoelasticity. Skin 
SOHAG MEDICAL JOURNAL

Vol. 25 No. 3 July 2021
An update in the etiopathogenesis of striae distensae Aya Ahmed Al-shandawely

and Reconstructive Surgery Global Open. 2021; 9(2): e3429.

28-Neinaa Y, Gheida S, and Mohamed D: Synergistic effect of platelet-rich plasma in combination with fractional carbon dioxide laser versus its combination with pulsed dye laser in striae distensae: A comparative study. Photodermatology, Photoimmunology \& Photomedicine. 2021; 37(3):214-23. 